\title{
A discrete-time scheduling model for continuous power-intensive process networks with various power contracts
}

\author{
Qi Zhang $^{\mathrm{a}}$, Arul Sundaramoorthy ${ }^{\mathrm{b}}$, Ignacio E. Grossmann ${ }^{\mathrm{a}, *}$, Jose M. Pinto ${ }^{\mathrm{c}}$ \\ ${ }^{a}$ Center for Advanced Process Decision-making, Department of Chemical Engineering, \\ Carnegie Mellon University, Pittsburgh, PA 15213, USA \\ ${ }^{b}$ Praxair, Inc., Business and Supply Chain Optimization RED, Tonawanda, NY 14150 \\ USA \\ ${ }^{c}$ Praxair, Inc., Business and Supply Chain Optimization RED, Danbury, CT 06810, USA
}

\begin{abstract}
Increased volatility in electricity prices and new emerging demand side management opportunities call for efficient tools for the optimal operation of powerintensive processes. In this work, a general discrete-time model is proposed for the scheduling of power-intensive process networks with various power contracts. The proposed model consists of a network of processes represented by Convex Region Surrogate models that are incorporated in a mode-based scheduling formulation, for which a block contract model is considered that allows the modeling of a large variety of commonly used power contracts. The resulting mixed-integer linear programming model is applied to an illustrative example as well as to a real-world industrial test case. The results demonstrate the model's capability in representing the operational flexibility in a process network and different electricity pricing structures. Moreover, because of its computational efficiency, the model holds much promise for its use in a real industrial setting.
\end{abstract}

Keywords: Production scheduling, demand side management, process networks, power contracts, mixed-integer linear programming

\section{Introduction}

With deregulated electricity markets and increasing penetration of intermittent renewable energy into the electricity supply mix, the level of uncertainty in the power grid has increased tremendously. This has led to highly volatile electricity prices, which pose immense challenges to power-intensive industries, such as air separation, aluminum, and chlor-alkali manufacturing. Demand side management (DSM), which refers to electric energy management on the consumers' side, has the potential of both significantly reducing the electricity cost

\footnotetext{
${ }^{*}$ Corresponding author

Email address: grossmann@cmu.edu (Ignacio E. Grossmann)
}

Preprint submitted to Elsevier

September 21, 2015

(C) 2015. This manuscript version is made available under the Elsevier user license

http://www.elsevier.com/open-access/userlicense/1.0/ 
for the consumer as well as improving the efficiency and reliability of the power grid.

Only in recent years, the high potential benefits of DSM for the chemical processing industry have been acknowledged by researchers and practitioners (Paulus and Borggrefe, 2011; Samad and Kiliccote, 2012; Merkert et al., 2014). One main concept of industrial DSM is to use the operational flexibility of the plant, which consumes a large amount of power, to take advantage of time-sensitive electricity prices (Charles River Assosicates, 2005; Albadi and El-Saadany, 2008). This can be achieved, for example, by shifting the production to low-price hours; however, load shifting has to be well-considered since one still has to satisfy process constraints and meet product demand. The critical time component in DSM calls for effective scheduling tools that consider the constraints on the production process as well as the price structures and purchasing limitations for different power sources.

Production scheduling has been an active area of research in process systems engineering (PSE) since the late 1970s. Since then, much progress has been made in the modeling of both batch and continuous scheduling problems as well as in the development of efficient methods for solving these models. Numerous general scheduling models have been proposed, many of which are based on the concepts of state-task network (STN) (Kondili et al., 1993; Shah et al., 1993) or resource-task network (RTN) (Pantelides, 1994). In this work, we focus on the specific case of continuous power-intensive production processes. Hence, for recent general reviews on the broad area of production scheduling in PSE, we refer to Méndez et al. (2006), Maravelias (2012), and Harjunkoski et al. (2014).

In recent years, scheduling frameworks for DSM have been proposed for various industrial power-intensive processes such as steelmaking (Ashok, 2006; Castro et al., 2013), electrolysis (Babu and Ashok, 2008), cement production (Vujanic et al., 2012), and air separation (Ierapetritou et al., 2002; Karwan and Keblis, 2007; Zhang et al., 2015a). In their comprehensive review, Zhang and Grossmann (2015) present an overview of the advances made in planning and scheduling for industrial DSM, and highlight future challenges in this area. One of the main research opportunities identified in the review is the integration of detailed production scheduling and energy management considering various purchasing options for electricity.

One important attribute of scheduling models is the representation of time, which is especially critical in DSM applications because of the highly timesensitive nature of electricity prices. In general, one distinguishes between discrete- and continuous-time models, and there is a large body of work in the literature discussing different formulations and their strengths and limitations (Floudas and Lin, 2004; Méndez et al., 2006; Sundaramoorthy and Maravelias, 2011). In a discrete time representation, in which the scheduling horizon is divided into discrete time periods, it is straightforward to model the time-varying electricity price by simply assigning different price values to different time periods. In a continuous time representation, achieving the same effect requires more complex constraints and usually leads to models that are much more difficult to solve for large-scale instances (Castro et al., 2009). 
In this work, we consider continuous production processes, for which numerous production scheduling models have been proposed in the literature. Some of the proposed models are based on the STN framework; see, for example, Ierapetritou and Floudas (1998), Giannelos and Georgiadis (2002), and Shaik and Floudas (2007). Many other models are based on the RTN framework, e.g. Zhang and Sargent (1996), Schilling and Pantelides (1996), and Castro et al. (2004). Some researchers have combined batch and continuous scheduling, or have focused on the case with parallel processes (Karimi and McDonald, 1997; Kopanos et al., 2011). Also, cyclic scheduling has been considered (Sahinidis and Grossmann, 1991; Pinto and Grossmann, 1994; Alle et al., 2004). Note that many of the recent research efforts have focused on the development of new continuous-time formulations.

Castro et al. $(2009,2011)$ present RTN-based discrete-time and continuoustime models for the scheduling of continuous plants under variable electricity cost. For the same purpose, Mitra et al. (2012) have developed a discrete-time scheduling model based on the concept of operating modes, which allows the systematic modeling of operational transitions. The resulting mixed-integer linear programming (MILP) model is computationally very efficient, and therefore allows the solution of large-scale industrial problems. However, in the proposed approach, a surrogate model is created for the plant as a whole, which does not explicitly consider interactions between different processes in the plant. Also, the model only considers day-ahead electricity prices and does not take purchasing electricity from pre-agreed power contracts into account.

In this work, we extend the model proposed by Mitra et al. (2012) and further generalize it such that it can be applied to continuous process networks subject to various power contracts. In order to achieve this goal, we explicitly model the process network, in which processes are connected by material flows, and each process is represented by a so-called Convex Region Surrogate (CRS) model. The mode-based scheduling formulation is applied as well as a block contract formulation to incorporate different types of power contracts.

The remainder of this paper is organized as follows. In Section 2, the problem statement is presented before the MILP model is developed in Section 3. The proposed model is applied to an illustrative example and an industrial air separation case study as shown in Sections 4 and 5, respectively. Finally, in Section 6 , we close with a summary of the results and concluding remarks.

\section{Problem statement}

We consider process networks involving continuous processes which consume significant amount of electric energy during operation. Here, a process can refer to a piece of equipment, a set of multiple interconnected pieces of equipment, or an entire plant. The processes in such a network differ in their feeds and products, the restrictions on the production rates, and the power consumption characteristics. Inventory capacities are given for storable intermediate and final products. Also, for all final products, the demand is given. 
Electricity can be purchased from various power contracts which may differ in price, availability, and penalty for under- or overconsumption. We assume that the prices and other conditions for negotiated contracts are known in advance, and that accurate forecasts for the day-ahead or real-time electricity prices are available.

The goal is to find a production schedule over a given time horizon that minimizes the total electricity cost while satisfying all product demand. For every time period of the scheduling horizon, we determine

- the mode of operation for each process,

- the processing rate in each process,

- the material flows in the process network,

- the amount of intermediate and final products stored,

- the amount of electricity purchased from each power contract.

\section{Model formulation}

We propose an MILP scheduling model, for which the mathematical formulation is presented below. Note that all continuous variables in this model are constrained to be nonnegative. A list of indices, sets, parameters, and variables is given in the Nomenclature section.

\subsection{Time representation}

A discrete-time framework is applied in which the time horizon is divided into time periods of equal length, $\Delta t$ (typically an hour). The notation for the time discretization is such that time period $t$ starts at time point $t-1$ and ends at time point $t$. The scheduling horizon is defined by the set of time periods $\bar{T}=\left\{1,2, \ldots, t^{\mathrm{fin}}\right\} . \bar{T}$ is a subset of $T=\left\{-\theta^{\max }+1,-\theta^{\max }+2, \ldots, 0,1, \ldots, t^{\mathrm{fin}}\right\}$, which also includes time periods in the past that are used in some constraints involving multiple time periods. The common-grid representation is illustrated in Figure 1.

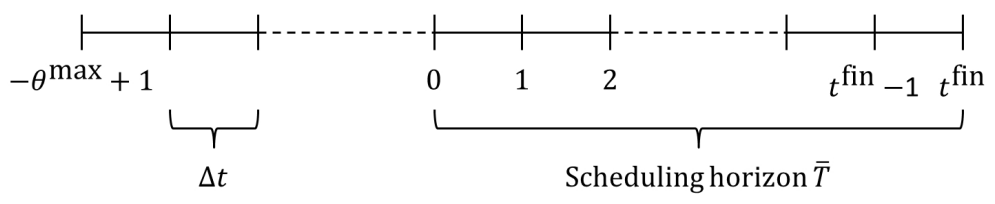

Figure 1: The proposed model applies a common-grid time representation with a time period length of $\Delta t$ and the present time point defined as time point 0 . 


\subsection{Process network representation}

We consider a process network consisting of processes that are connected by material nodes, which represent potential storage units for feeds, intermediate products, and final products. The arcs in such a network depict the directions of the material flows. Two or more streams entering the same material node are of the same quality, i.e. same chemical, temperature, pressure, etc., similarly as in the STN model (Kondili et al., 1993). Figure 2 shows an example of such a process network, in which the process and material nodes are denoted by rectangles and circles, respectively.

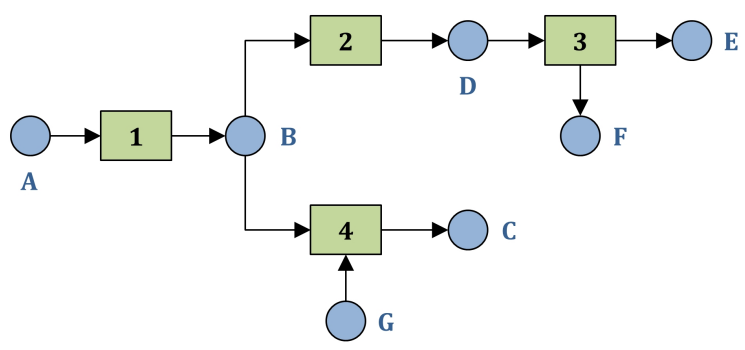

Figure 2: This example shows a simple process network consisting of process nodes (rectangles), material nodes (circles), and arcs depicting the material flows.

\subsection{Mass balance constraints}

For a given process network operating continuously in each time interval $t$, the mass balance constraints can be stated as follows:

$$
\begin{array}{ll}
Q_{j t}=Q_{j, t-1}+\sum_{i \in \hat{I}_{j}} P_{i j t}-\sum_{i \in \bar{I}_{j}} P_{i j t}+W_{j t}-D_{j t} & \forall j, t \in \bar{T} \\
Q_{j}^{\min } \leq Q_{j t} \leq Q_{j}^{\max } & \forall j, t \in \bar{T} \\
W_{j t} \leq W_{j}^{\max } & \forall j, t \in \bar{T}
\end{array}
$$

where $Q_{j t}$ is the inventory level for material $j$ at time $t$, and $P_{i j t}$ is the amount of material $j$ consumed or produced by process $i$ in time period $t$. $\hat{I}_{j}$ denotes the set of processes producing material $j$, whereas $\bar{I}_{j}$ is the set of processes receiving material $j$. The additional purchase of material $j$ in time period $t$ is denoted by $W_{j t}$. Parameter $D_{j t}$ denotes the demand for material $j$ in time period $t$. Eq. (1a) states that the inventory level of a material increases when it is produced or purchased, and the inventory level decreases when the material is consumed by other processes or used to meet product demand. Eq. (1b) sets lower and upper bounds on the inventory levels. For nonstorable materials, $Q_{j}^{\min }$ and $Q_{j}^{\max }$ are zero. Eq. (1c) limits the amount of material that can be purchased per time period. 


\subsection{Process surrogate model}

In addition to the mass balance constraints that reflect the structure of the process network, we require a model for each individual process that represents its input-output relationship and expresses its capacity. Furthermore, for every feasible operating point, we have to know the amount of electricity required so that it can be included in the objective function. In other words, a description of the feasible operating region of each process is required in the space of its input and output materials, and an electricity consumption correlation that is a function of those inputs and outputs.

In this framework, it is assumed that each process can operate in different operating modes. Each mode represents a particular operating state, e.g. "off", "on", or "startup". In the proposed model, the feasible operating region for each mode is defined by a union of convex subregions in the corresponding material space, and a linear electricity consumption function with respect to the production rates is given for each subregion. The key feature here is that every subregion has the form of a polytope. Figure 3 shows an illustrative example of the feasible operating region of a process in a two-dimensional material space. In this case, the materials are P1 and P2, and the process can operate in three different modes, Modes 1, 2, and 3. The feasible space of Mode 1 is a single point at the origin, which denotes zero production; thus, this mode is the "off" mode. The feasible space of Mode 2 is described by one single polytope, while Mode 3 is captured by the union of two polytopes, $3 \mathrm{a}$ and $3 \mathrm{~b}$. Note that the feasible regions of different modes may overlap as it is the case here for Modes 2 and 3 .

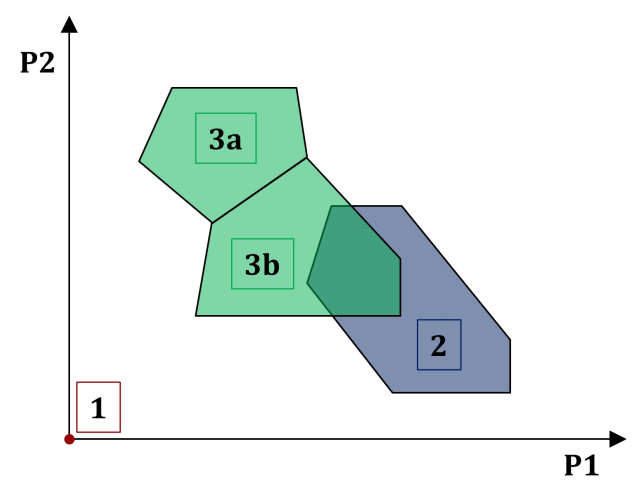

Figure 3: This illustrative example shows the feasible operating region of a process that can operate in three different operating modes.

Obviously, the feasible region of a real process typically does not have this polyhedral form. Usually, this is an approximation of the true feasible region. However, as it will be shown in the following, this representation allows us to formulate models for each process in a uniform manner, which are computationally efficient when embedded in an MILP scheduling model. Such a model is 
generally referred to as a Convex Region Surrogate (CRS) model. For complex processes, CRS models can be constructed by either using a model-based (Sung and Maravelias, 2009) or a data-driven approach (Zhang et al., 2015b).

Physically, at any point in time, a process can only run in one operating mode. For a given operating mode, the operating point has to lie in either one of the convex subregions. Any point in a subregion can be represented as a convex combination of the vertices of the polytope. These relationships can be expressed by the following nested disjunction:

$$
\begin{aligned}
& \bigvee_{m \in M_{i}}\left[\bigvee_{r \in R_{i m}}\left(\begin{array}{c}
Y_{i m t} \\
\bar{Y}_{i m r t} \\
P_{i j t}=\sum_{l \in L_{i m r}} \lambda_{i m r l t} \phi_{i m r l j} \quad \forall j \in J_{i} \\
\sum_{l \in L_{i m r}} \lambda_{i m r l t}=1 \\
0 \leq \lambda_{i m r l t} \leq 1 \quad \forall l \in L_{i m r} \\
U_{i t}=\delta_{i m r}+\sum_{j \in J_{i}} \gamma_{i m r j} P_{i j t}
\end{array}\right)\right] \forall i, t \in \bar{T} \\
& \underset{m \in M_{i}}{\vee} Y_{i m t} \quad \forall i, t \in \bar{T} \\
& Y_{i m t} \Leftrightarrow \underset{r \in R_{i m}}{\frac{\vee}{R_{i m r t}}} \quad \forall i, m \in M_{i}, t \in \bar{T} \\
& Y_{\text {imt }} \in\{\text { true, false }\} \quad \forall i, m \in M_{i}, t \in \bar{T} \\
& \bar{Y}_{i m r t} \in\{\text { true, false }\} \quad \forall i, m \in M_{i}, r \in R_{i m}, t \in \bar{T}
\end{aligned}
$$

where $M_{i}$ is the set of modes in which process $i$ can operate, $R_{i m}$ is the set of operating subregions in mode $m \in M_{i}, L_{i m r}$ is the set of vertices of subregion $r \in R_{i m}$, and $J_{i}$ is the set of input and output materials of process $i$. $Y_{i m t}$ and $\bar{Y}_{i m r t}$ are boolean variables. $Y_{i m t}$ is true if mode $m \in M_{i}$ is selected in time period $t$, whereas $\bar{Y}_{i m r t}$ is true if subregion $r \in R_{i m}$ is selected in time period $t$. The argument of the inner disjunction in (2a) states that the amount of material $j \in J_{i}$ consumed or produced by process $i, P_{i j t}$, is expressed as a convex combination of the corresponding vertices, $\phi_{i m r l j}$, while the amount of electricity consumed, $U_{i t}$, is a linear function of $P_{i j t}$ with a constant $\delta_{i m r}$ and coefficients $\gamma_{i m r j}$ specific to the selected subregion. Eq. (2b) states that one and only one mode has to be selected for each process in each time period, and according to Eq. (2c), one region in $R_{i m}$ has to be selected if process $i$ operates in mode $m \in M_{i}$.

By applying the hull reformulation (Balas, 1985), the disjunction given by Eqs. (2) can be transformed into the following set of mixed-integer linear constraints:

$$
\begin{aligned}
& P_{i j t}=\sum_{m \in M_{i}} \sum_{r \in R_{i m}} \bar{P}_{i m r j t} \quad \forall i, j \in J_{i}, t \in \bar{T} \\
& \bar{P}_{\text {imrjt }}=\sum_{l \in L_{i m r}} \lambda_{\text {imrlt }} \phi_{\text {imrlj }} \quad \forall i, m \in M_{i}, r \in R_{i m}, j \in J_{i}, t \in \bar{T}
\end{aligned}
$$




$$
\begin{aligned}
& \sum_{l \in L_{i m r}} \lambda_{i m r l t}=\bar{y}_{i m r t} \quad \forall i, m \in M_{i}, r \in R_{i m}, t \in \bar{T} \\
& U_{i t}=\sum_{m \in M_{i}} \sum_{r \in R_{i m}}\left(\delta_{i m r} \bar{y}_{i m r t}+\sum_{j \in J_{i}} \gamma_{i m r j} \bar{P}_{i m r j t}\right) \quad \forall i, t \in \bar{T} \\
& y_{i m t}=\sum_{r \in R_{i m}} \bar{y}_{i m r t} \quad \forall i, m \in M_{i}, t \in \bar{T} \\
& \sum_{m \in M_{i}} y_{i m t}=1 \quad \forall i, t \in \bar{T}
\end{aligned}
$$

where $y_{i m t}$ and $\bar{y}_{i m r t}$ are binary variables, and $\bar{P}_{i m r j t}$ is the disaggregated variable. For each $i, j \in J_{i}, t \in \bar{T}$, only one $\bar{P}_{\text {imrjt }}$ can be nonzero. Note that an equivalent formulation is achieved if $y_{i m t}$ is relaxed to be a continuous variable with $0 \leq y_{\text {imt }} \leq 1$ since Eq. (3e) forces it to take either the value zero or one.

\subsection{Transition constraints}

A transition occurs when the system changes from one operating point to another. For changes between operating points belonging to the same operating mode, a bound on the rate of change, $\Delta_{i m j}^{\max }$, can be set with Eq. (4).

$$
-\Delta_{i m j}^{\max } \leq \sum_{r \in R_{i m}}\left(\bar{P}_{i m r j t}-\bar{P}_{i m r j, t-1}\right) \leq \Delta_{i m j}^{\max } \quad \forall i, m \in M_{i}, j \in J_{i}, t \in \bar{T}
$$

Additional constraints have to be imposed on transitions between different modes. The dynamics of a process in terms of its mode transition behavior can be visualized with a mode transition graph. Figure 4 shows an example with four different operating modes: off, on, startup, and shutdown. The arcs in the graph show the directions of the allowed transitions with the corresponding operational constraints. For instance, after shutting down the process, i.e. transitioning from the shutdown to the off mode, the process has to remain for at least 24 hours in the off mode before it can move to the startup mode. This constraint is typically imposed to reduce stress on the equipment. According to the mode transition graph, the startup phase takes exactly 4 hours before the process moves into its normal production mode, in which it has to remain for at least 48 hours.

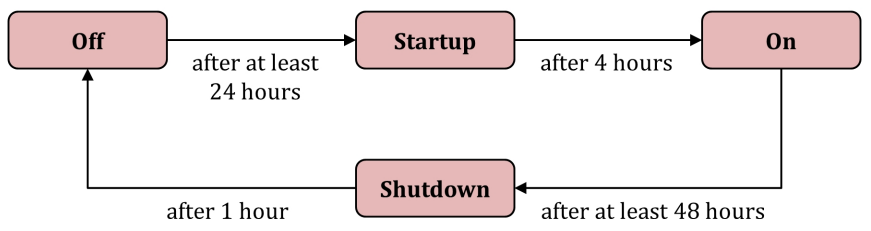

Figure 4: The mode transition graph shows the different operating modes and all possible transitions with the corresponding operational constraints. 
Eqs. (5)-(7), which model the transition constraints, are adopted from Mitra et al. $(2012,2013)$. Here, $z_{i m m^{\prime} t}$ is a binary variable which is 1 if and only if process $i$ switches from mode $m$ to mode $m^{\prime}$ at time $t$, which is enforced by the following constraint:

$$
\sum_{m^{\prime} \in T R_{i m}^{\mathrm{f}}} z_{i m^{\prime} m, t-1}-\sum_{m^{\prime} \in T R_{i m}^{\mathrm{t}}} z_{i m m^{\prime}, t-1}=y_{i m t}-y_{i m, t-1} \quad \forall i, m \in M_{i}, t \in \bar{T}
$$

where $T R_{i m}^{\mathrm{f}}=\left\{m^{\prime}:\left(m^{\prime}, m\right) \in T R_{i}\right\}$ and $T R_{i m}^{\mathrm{t}}=\left\{m^{\prime}:\left(m, m^{\prime}\right) \in T R_{i}\right\}$ with $T R_{i}$ being the set of all possible mode-to-mode transitions for process $i$.

The restriction that a process has to remain in a certain mode for a minimum amount of time after a transition is expressed by the following constraint:

$$
y_{i m^{\prime} t} \geq \sum_{k=1}^{\theta_{i m m^{\prime}}} z_{i m m^{\prime}, t-k} \quad \forall i,\left(m, m^{\prime}\right) \in T R_{i}, t \in \bar{T}
$$

with $\theta_{i m m^{\prime}}$ being the minimum stay time in mode $m^{\prime}$ after switching to it from mode $m$.

For predefined sequences, each defined as a fixed chain of transitions from mode $m$ to mode $m^{\prime}$ to mode $m^{\prime \prime}$, we can specify a fixed stay time in mode $m^{\prime}$ by imposing the following constraint:

$$
z_{i m m^{\prime}, t-\bar{\theta}_{i m m^{\prime} m^{\prime \prime}}}=z_{i m^{\prime} m^{\prime \prime} t} \quad \forall i,\left(m, m^{\prime}, m^{\prime \prime}\right) \in S Q_{i}, t \in \bar{T}
$$

where $S Q_{i}$ is the set of predefined sequences and $\bar{\theta}_{i m m^{\prime} m^{\prime \prime}}$ is the fixed stay time in mode $m^{\prime}$ in the corresponding sequence. For instance, in the example shown in Figure 4, this constraint applies to the sequence off $\rightarrow$ startup $\rightarrow$ on. Since the startup process takes a certain amount of time, we can use Eq. (7) to fix the number of time periods in which the process has to remain in this mode once selected.

\subsection{Energy balance constraints}

The required amount of electricity can be purchased from multiple sources, which is expressed in Eq. (8a). The different power sources are available power contracts, denoted by index $c$. Eq. (8b) sets lower and upper bounds on the electricity purchase from contract $c$ in time period $t, E_{c t}$. The lower bound, $E_{c t}^{\mathrm{min}}$, is typically zero, but could be nonzero if the contract conditions demand a minimum purchase.

$$
\begin{array}{ll}
\sum_{i} U_{i t}=\sum_{c} E_{c t} & \forall t \in \bar{T} \\
E_{c t}^{\min } \leq E_{c t} \leq E_{c t}^{\max } & \forall c, t \in \bar{T}
\end{array}
$$

\subsection{Power contract model}

Eqs. (8) are sufficient if power is only purchased from the spot market or from contracts that are merely defined by a unit price for each time period and 
possibly some minimum and maximum purchasing restrictions. However, large industrial electricity consumers typically commit themselves to power contracts that provide additional favorable conditions. There are a large variety of such power contract structures; in this model, we consider the two most common types: discount and penalty contracts. With a discount contract, the unit price decreases with increasing amount of purchased electricity. For penalty contracts, the consumer agrees to either purchase at least a certain amount of electricity and pay a penalty for underconsumption, or not exceed a certain amount to avoid penalty for overconsumption.

Discount prices and penalties are defined with respect to the amount of electricity purchased over a certain period of time, which could be hours, days, or even weeks. In practice, this means that the cumulative electricity purchase is recorded, and there are predefined meter reading times at which the amount of electricity purchased since the last meter reading is computed. According to this cumulative electricity purchase between consecutive meter readings, discount prices and penalties are issued. In our model, we track the cumulative electricity purchase by using the following equations:

$$
\begin{array}{ll}
F_{c t}=F_{c, t-1}+E_{c t} & \forall c \in \bar{C}, t \in \bar{T} \backslash \widehat{T}_{c} \\
F_{c t}=F_{c, t-1}+E_{c t}-G_{c t} & \forall c \in \bar{C}, t \in \widehat{T}_{c} \\
F_{c t}=0 & \forall c \in \bar{C}, t \in\left\{0, \widehat{T}_{c}\right\}
\end{array}
$$

where $\bar{C}$ is the set of discount and penalty contracts and $\widehat{T}_{c}$ is the set of meter reading times for contract $c$, which does not include time $0 . F_{c t}$ denotes the cumulative electricity purchase, which is reset to zero at every meter reading time. We further introduce the variable $G_{c t}$, which is the cumulative electricity purchase since the last meter reading before time $t$. To further clarify the notation, Figure 5 shows an illustrative example in which the cumulative electricity purchase meter readings are conducted every four time periods.

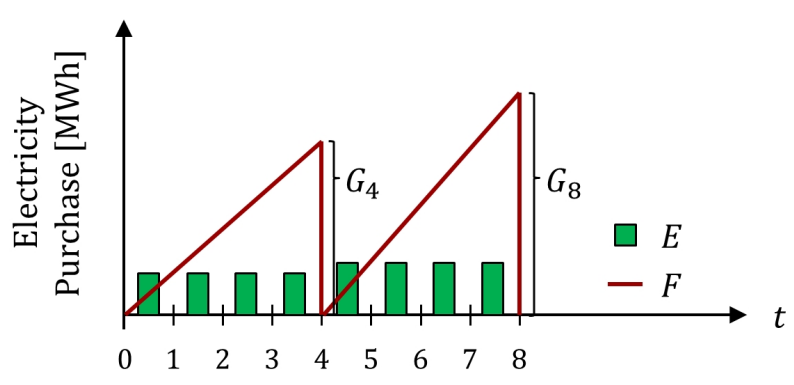

Figure 5: In this illustrative example, the cumulative electricity purchase meter is read every four hours.

To model discount and penalty contracts, we develop a block contract formulation that can accommodate both types of contracts. In a general block contract, each block is defined by an electricity price and the corresponding 
amount of electricity that one has to purchase in order to reach this block. In the case of a discount contract, the unit price decreases with each block as illustrated in Figure 6.

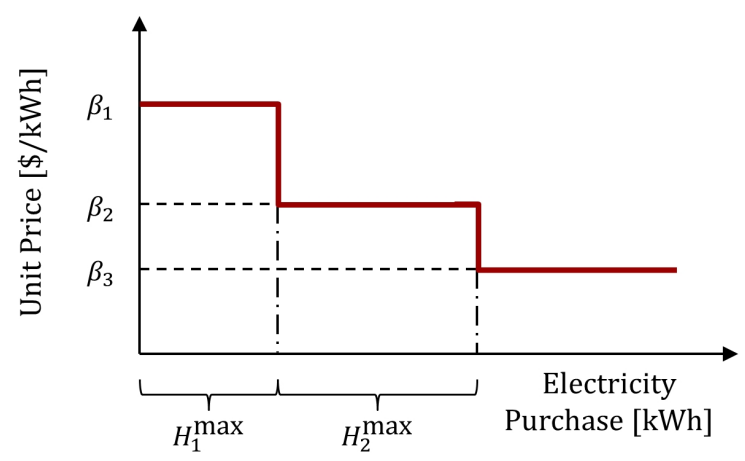

Figure 6: With a discount contract, unit price decreases with increasing electricity purchase. The ranges for the different prices define the contract blocks.

The following disjunction expresses the mechanism of a block contract:

$$
\begin{array}{ll}
\bigvee_{b \in B_{c}}\left[\begin{array}{cc}
X_{c b t} \\
H_{c b^{\prime} t}=H_{c b^{\prime}}^{\max } & \forall b^{\prime} \in B_{c}, b^{\prime}<b \\
H_{c b} \leq H_{c b}^{\max } & \\
H_{c b^{\prime} t}=0 \quad \forall b^{\prime} \in B_{c}, b^{\prime}>b
\end{array}\right] & \forall c \in \bar{C}, t \in \widehat{T}_{c} \\
\frac{\vee}{b \in B_{c}} & \forall c \in \bar{C}, t \in \widehat{T}_{c} \\
X_{c b t} \in\{\text { true, false }\} & \forall c \in \bar{C}, b \in B_{c}, t \in \widehat{T}_{c}
\end{array}
$$

where $B_{c}$ is the set of blocks for contract $c, H_{c b t}$ denotes the amount of cumulative electricity purchased in block $b \in B_{c}$ at time $t$, and $H_{c b}^{\max }$ is the amount of electricity that one has to purchase in block $b \in B_{c}$ before reaching the next block. $X_{c b t}$ is a boolean variable that is true if block $b$ is the highest block reached for contract $c$ at time $t$. Disjunction (10a) states that if $X_{c b t}$ is true, the maximum amount is purchased in all lower blocks $b^{\prime}<b$, the electricity purchase in block $b$ is bounded by $H_{c b}^{\max }$, and no electricity is purchased in higher blocks $b^{\prime}>b$. According to logic constraint (10b), one and only one $X_{c b t}$ has to be true.

Again, by applying the hull reformulation, Eqs. (10) can be transformed into the following mixed-integer linear constraints:

$$
\begin{array}{ll}
\sum_{b \in B_{c}} x_{c b t}=1 & \forall c \in \bar{C}, t \in \widehat{T}_{c} \\
\bar{H}_{c b^{\prime} b t}=H_{c b^{\prime}}^{\max } x_{c b t} & \forall c \in \bar{C}, b \in B_{c}, b^{\prime} \in B_{c}, b^{\prime}<b, t \in \widehat{T}_{c} \\
\bar{H}_{c b b t} \leq H_{c b}^{\max } x_{c b t} & \forall c \in \bar{C}, b \in B_{c}, t \in \widehat{T}_{c}
\end{array}
$$




$$
H_{c b t}=\sum_{b^{\prime} \in B_{c}, b^{\prime} \geq b} \bar{H}_{c b b^{\prime} t} \quad \forall c \in \bar{C}, b \in B_{c}, t \in \widehat{T}_{c}
$$

where $x_{c b t}$ is a binary variable, and $\bar{H}_{c b b^{\prime} t}$ is the disaggregated variable.

To obtain the amount of electricity purchased from a contract, we sum up the electricity purchased in all corresponding contract blocks, as stated in Eq. (12).

$$
G_{c t}=\sum_{b \in B_{c}} H_{c b t} \quad \forall c \in \bar{C}, t \in \widehat{T}_{c}
$$

We can use the same block contract formulation to model penalty contracts by specifying $H_{c b}^{\max }$ such that the first block (Block 1) and the last block (Block $\left.\left|B_{c}\right|\right)$ correspond to under- and overconsumption, respectively. A simple penalty contract is illustrated in Figure 7. Here, if $H_{c b t}<H_{c b}^{\max }$ for $b=1$, we underconsume, whereas if $H_{c b t}>0$ for $b=3$, we overconsume.

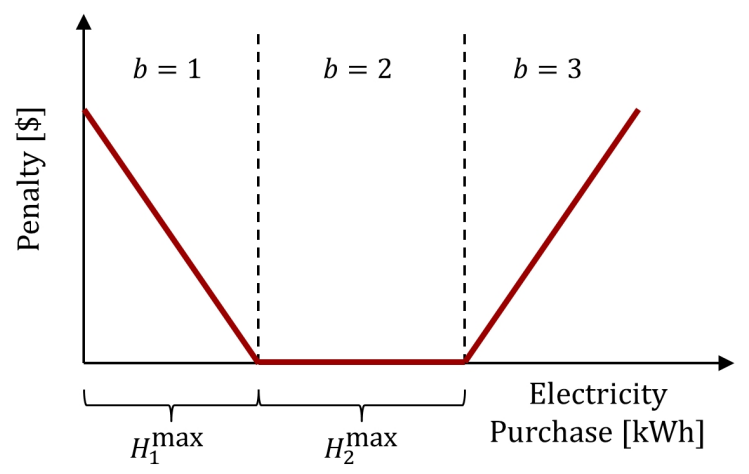

Figure 7: A simple penalty contract can be modeled as a three-block contract, with the first and third blocks corresponding to under- and overconsumption, respectively.

\subsection{Boundary conditions}

We solve the scheduling problem for a given time horizon. For the problem to be well-defined, boundary conditions are required. The following initial conditions set the initial inventory levels, the initial operating modes, and the mode switching history:

$$
\begin{array}{ll}
Q_{j, 0}=Q_{j}^{\mathrm{ini}} & \forall j \\
y_{i m, 0}=y_{i m}^{\mathrm{ini}} & \forall i, m \in M_{i} \\
z_{i m m^{\prime} t}=z_{i m m^{\prime} t}^{\mathrm{ini}} & \forall i,\left(m, m^{\prime}\right) \in T R_{i},-\theta_{i}^{\max }+1 \leq t \leq-1
\end{array}
$$

with $\theta_{i}^{\max }=\max \left(\max _{\left(m, m^{\prime}\right) \in T R_{i}}\left\{\theta_{i m m^{\prime}}\right\}, \max _{\left(m, m^{\prime}, m^{\prime \prime}\right) \in S Q_{i}}\left\{\bar{\theta}_{i m m^{\prime} m^{\prime \prime}}\right\}\right)$, which defines for how far back in the past the mode switching information has to be provided. 
In the following terminal constraints, we simply set lower bounds on the final inventory levels.

$$
Q_{j, t^{\mathrm{fin}}} \geq Q_{j}^{\mathrm{fin}} \quad \forall j
$$

\subsection{Objective function}

The objective is to minimize the total electricity cost, $T C$, as expressed in the following objective function:

$$
T C=\sum_{c} \sum_{t \in \bar{T}} \alpha_{c t} E_{c t}+\sum_{c \in \bar{C}} \sum_{t \in \widehat{T}_{c}}\left[\sum_{b \in B_{c}} \beta_{c b t} H_{c b t}+\zeta_{c t}^{\mathrm{u}}\left(H_{c, 1}^{\max }-H_{c, 1, t}\right)+\zeta_{c t}^{\mathrm{o}} H_{c,\left|B_{c}\right|, t}\right]
$$

where $\alpha_{c t}$ and $\beta_{c b t}$ are unit costs for purchased electricity, whereas $\zeta_{c t}^{\mathrm{u}}$ and $\zeta_{c t}^{\mathrm{o}}$ are unit penalty costs for under- and overconsumption, respectively. The first term in Eq. (15) represents the base cost and applies to all contracts while the remaining terms only apply to block contracts.

Note that the price structure of a contract is defined by the cost coefficients $\alpha_{c t}, \beta_{c b t}, \zeta_{c t}^{\mathrm{u}}, \zeta_{c t}^{\mathrm{o}}$, as well as $H_{c b}^{\max }$. Many combinations are possible, which provides the flexibility of modeling various different power contracts. Some common examples are listed in the following:

- Day-ahead or real-time market: $\alpha_{c t}>0, \beta_{c b t}=0, \zeta_{c t}^{\mathrm{u}}=0, \zeta_{c t}^{\mathrm{o}}=0$

- Pure discount contract: $\alpha_{c t}=0, \beta_{c b t}>0, \zeta_{c t}^{\mathrm{u}}=0, \zeta_{c t}^{\mathrm{o}}=0$

- Contract with partial price discount: $\alpha_{c t}>0, \beta_{c b t}>0, \zeta_{c t}^{\mathrm{u}}=0, \zeta_{c t}^{\mathrm{o}}=0$

- Contract with penalty for underconsumption: $\alpha_{c t}>0, \beta_{c b t}=0, \zeta_{c t}^{\mathrm{u}}>0$, $\zeta_{c t}^{\mathrm{o}}=0$

- Contract with penalty for overconsumption: $\alpha_{c t}>0, \beta_{c b t}=0, \zeta_{c t}^{\mathrm{u}}=0$, $\zeta_{c t}^{\mathrm{o}}>0$

- Contract with penalties for under- and overconsumption: $\alpha_{c t}>0, \beta_{c b t}=0$, $\zeta_{c t}^{\mathrm{u}}>0, \zeta_{c t}^{\mathrm{o}}>0$

- Combined discount and penalty contract: $\alpha_{c t}>0, \beta_{c b t}>0, \zeta_{c t}^{\mathrm{u}}>0, \zeta_{c t}^{\mathrm{o}}>0$

By considering the objective function given by Eq. (15), it is assumed that electricity cost constitutes the vast majority of the operating cost or that other costs required to satisfy the given demand are constant. If this assumption is invalid and additional costs - such as inventory costs, mode transition costs, and costs of purchasing material - need to be considered, they can be easily incorporated by assigning cost coefficients to the corresponding variables. 


\section{Illustrative example}

To demonstrate the main features of the model, we first apply it to an illustrative example for which the process network is shown in Figure 8.

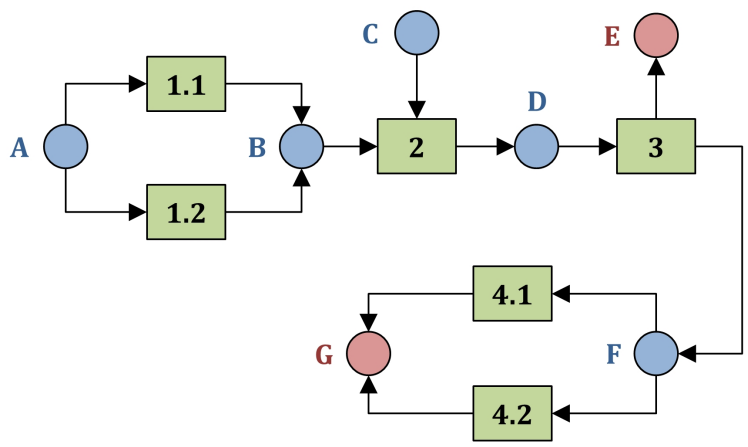

Figure 8: The process network of the illustrative example consists of four process and seven material nodes.

Each process is characterized by its operating modes and operating subregions. The vertices of each convex subregion are listed in Table 1 where each vertex is given as a vector of the materials. Note that there is a different set of vertices for each subregion; however, in every vertex set, the numbering of the vertices starts at 1 . Also, notice that the on mode of Process 3 is the only mode that is described by two subregions. Each operating subregion is further characterized by a linear electricity consumption function, which is shown in Table 2. Possible transitions between different operating modes and predefined sequences are listed in Tables 3 and 4, respectively; the tables also contain the corresponding minimum and fixed stay times given in hours. No bound is imposed on the rate of change in the same mode. 
Table 1: Vertices associated with each operating subregion of the processes from the illustrative example.

\begin{tabular}{|c|c|c|c|c|c|c|c|c|c|c|}
\hline Process & Mode & Region & Vertex & $\mathbf{A}$ & B & $\mathrm{C}$ & $\mathrm{D}$ & $\mathbf{E}$ & $\mathbf{F}$ & $G$ \\
\hline \multirow{3}{*}{1.1} & off & 1 & 1 & 0 & 0 & 0 & 0 & 0 & 0 & 0 \\
\hline & \multirow{2}{*}{ on } & \multirow{2}{*}{1} & 1 & 60 & 60 & 0 & 0 & 0 & 0 & 0 \\
\hline & & & 2 & 600 & 600 & 0 & 0 & 0 & 0 & 0 \\
\hline \multirow{3}{*}{1.2} & off & 1 & 1 & 0 & 0 & 0 & 0 & 0 & 0 & 0 \\
\hline & \multirow{2}{*}{ on } & \multirow{2}{*}{1} & 1 & 20 & 20 & 0 & 0 & 0 & 0 & 0 \\
\hline & & & 2 & 200 & 200 & 0 & 0 & 0 & 0 & 0 \\
\hline \multirow{5}{*}{2} & off & 1 & 1 & 0 & 0 & 0 & 0 & 0 & 0 & 0 \\
\hline & startup & 1 & 1 & 0 & 0 & 0 & 0 & 0 & 0 & 0 \\
\hline & shutdown & 1 & 1 & 0 & 0 & 0 & 0 & 0 & 0 & 0 \\
\hline & \multirow{2}{*}{ on } & \multirow{2}{*}{1} & 1 & 0 & 40 & 20 & 60 & 0 & 0 & 0 \\
\hline & & & 2 & 0 & 800 & 4000 & 1200 & 0 & 0 & 0 \\
\hline \multirow{11}{*}{3} & off & 1 & 1 & 0 & 0 & 0 & 0 & 0 & 0 & 0 \\
\hline & startup & 1 & 1 & 0 & 0 & 0 & 0 & 0 & 0 & 0 \\
\hline & shutdown & 1 & 1 & 0 & 0 & 0 & 0 & 0 & 0 & 0 \\
\hline & \multirow{8}{*}{ on } & \multirow{4}{*}{1} & 1 & 0 & 0 & 0 & 100 & 50 & 50 & 0 \\
\hline & & & 2 & 0 & 0 & 0 & 100 & 45 & 55 & 0 \\
\hline & & & 3 & 0 & 0 & 0 & 1000 & 500 & 500 & 0 \\
\hline & & & 4 & 0 & 0 & 0 & 1000 & 450 & 550 & 0 \\
\hline & & \multirow{4}{*}{2} & 1 & 0 & 0 & 0 & 100 & 45 & 55 & 0 \\
\hline & & & 2 & 0 & 0 & 0 & 100 & 40 & 60 & 0 \\
\hline & & & 3 & 0 & 0 & 0 & 1000 & 450 & 550 & 0 \\
\hline & & & 4 & 0 & 0 & 0 & 1000 & 400 & 600 & 0 \\
\hline \multirow{3}{*}{4.1} & off & 1 & 1 & 0 & 0 & 0 & 0 & 0 & 0 & 0 \\
\hline & \multirow{2}{*}{ on } & \multirow{2}{*}{1} & 1 & 0 & 0 & 0 & 0 & 0 & 30 & 30 \\
\hline & & & 2 & 0 & 0 & 0 & 0 & 0 & 300 & 300 \\
\hline \multirow{3}{*}{4.2} & off & 1 & 1 & 0 & 0 & 0 & 0 & 0 & 0 & 0 \\
\hline & \multirow{2}{*}{ on } & \multirow{2}{*}{1} & 1 & 0 & 0 & 0 & 0 & 0 & 30 & 30 \\
\hline & & & 2 & 0 & 0 & 0 & 0 & 0 & 300 & 300 \\
\hline
\end{tabular}


Table 2: Electricity consumption correlations associated with each operating subregion. Each correlation is a linear function of the materials.

\begin{tabular}{cccc}
\hline Process & Mode & Region & $\begin{array}{c}\text { Electricity Consumption } \\
\text { Correlation }\end{array}$ \\
\hline \multirow{2}{*}{1.1} & off & 1 & 0 \\
& on & 1 & $500+2 \mathrm{~A}$ \\
\hline \multirow{2}{*}{1.2} & off & 1 & 0 \\
& on & 1 & $450+3 \mathrm{~A}$ \\
\hline \multirow{2}{*}{2} & off & 1 & 0 \\
& startup & 1 & 200 \\
& shutdown & 1 & 150 \\
& on & 1 & $100+0.5 \mathrm{D}$ \\
\multirow{4}{*}{3} & off & 1 & 0 \\
& startup & 1 & 200 \\
& shutdown & 1 & $800+3 \mathrm{D}$ \\
& on & 1 & $1000+4 \mathrm{D}$ \\
\hline \multirow{2}{*}{4.1} & on & 2 & 0 \\
& off & 1 & $400+2 \mathrm{~F}$ \\
\hline \multirow{2}{*}{4.2} & on & 1 & $350+1.5 \mathrm{~F}$ \\
\hline
\end{tabular}


Table 3: Possible transitions between the different operating modes of each process and the corresponding minimum stay times.

\begin{tabular}{ccc}
\hline Process & $\begin{array}{c}\text { Transition from } \\
\text { Mode } \boldsymbol{m} \text { to Mode } \boldsymbol{m}^{\prime}\end{array}$ & $\begin{array}{c}\text { Minimum Stay } \\
\text { Time in Mode } \boldsymbol{m}^{\prime}\end{array}$ \\
\hline \multirow{2}{*}{1.1} & off $\rightarrow$ on & 0 \\
& on $\rightarrow$ off & 0 \\
\hline \multirow{2}{*}{1.2} & off $\rightarrow$ on & 0 \\
& on $\rightarrow$ off & 0 \\
\hline \multirow{4}{*}{2} & off $\rightarrow$ startup & 1 \\
& startup $\rightarrow$ on & 6 \\
& on $\rightarrow$ shutdown & 1 \\
& shutdown $\rightarrow$ off & 6 \\
\hline \multirow{3}{*}{3} & off $\rightarrow$ startup & 2 \\
& startup $\rightarrow$ on & 6 \\
& on $\rightarrow$ shutdown & 1 \\
\multirow{2}{*}{4.1} & shutdown $\rightarrow$ off & 12 \\
\hline \multirow{2}{*}{4.2} & off $\rightarrow$ on & 0 \\
& on $\rightarrow$ off & 0 \\
\hline
\end{tabular}

Table 4: Predefined sequences of mode transitions and the corresponding fixed stay times.

\begin{tabular}{ccc}
\hline Process & $\begin{array}{c}\text { Sequence }(\text { Transition } \\
\left.\text { from } \boldsymbol{m} \text { to } \boldsymbol{m}^{\prime} \text { to } \boldsymbol{m}^{\prime \prime}\right)\end{array}$ & $\begin{array}{c}\text { Fixed Stay } \\
\text { Time in } \boldsymbol{m}^{\prime}\end{array}$ \\
\hline \multirow{2}{*}{2} & off $\rightarrow$ startup $\rightarrow$ on & 1 \\
& on $\rightarrow$ shutdown $\rightarrow$ off & 1 \\
\hline \multirow{2}{*}{3} & off $\rightarrow$ startup $\rightarrow$ on & 2 \\
& on $\rightarrow$ shutdown $\rightarrow$ off & 1 \\
\hline
\end{tabular}

Data regarding the inventory of each material are given in Table 5. Infinite availability of feedstock, A and C, is assumed. Also note that Intermediate B is not storable. Moreover, no material can be purchased during the scheduling horizon, i.e. $W_{j}^{\max }=0 \forall j$. 
Table 5: Inventory bounds and initial inventory levels for each material.

\begin{tabular}{cccccccc}
\hline & A & B & C & D & E & F & G \\
\hline Minimum Inventory & 0 & 0 & 0 & 0 & 3,000 & 0 & 5,000 \\
Maximum Inventory & $\infty$ & 0 & $\infty$ & 8,000 & 20,000 & 2,000 & 30,000 \\
Initial Inventory & $\infty$ & 0 & $\infty$ & 800 & 5,000 & 0 & 8,000 \\
Minimum Final Inventory & 0 & 0 & 0 & 800 & 5,000 & 0 & 8,000 \\
\hline
\end{tabular}

We consider a two-day scheduling horizon with an hourly time discretization. The results for three different product demand scenarios (low, high, and medium) will be compared; the corresponding demand profiles are shown in Figure 9. Note that in the low and high demand scenarios, the demands for E and $\mathrm{G}$ are constant over time while they vary in the medium demand scenario.

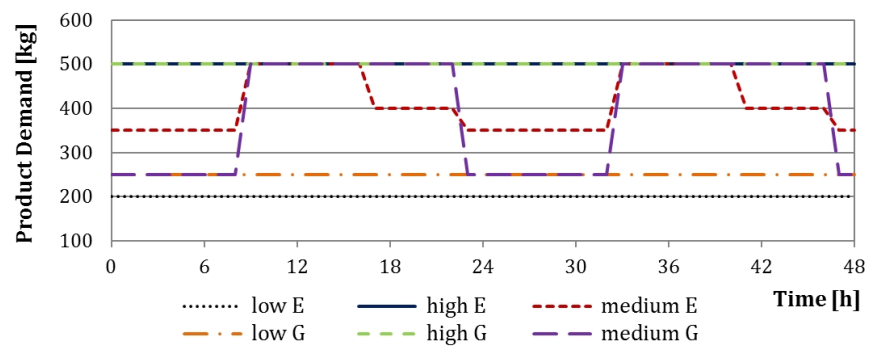

Figure 9: Product demand profiles for the three different scenarios (low, high, and medium).

Three different power contracts are considered: a time-of-use (TOU), a discount, and a penalty contract. In addition to different base electricity price profiles, other features differentiating the three contracts are the following:

- The TOU contract does not have any additional price components.

- With the discount contract, the additional cost depends on the purchased amount of electricity over the course of each day, i.e. the cumulative electricity consumption meter is read at the end of each day. For the first $50 \mathrm{MWh}$, an additional cost of $\$ 10 / \mathrm{MWh}$ needs to be paid; for the next $40 \mathrm{MWh}$, a cost of $\$ 8 / \mathrm{MWh}$ occurs; and for any amount of electricity purchased beyond $90 \mathrm{MWh}$, the cost is $\$ 5 / \mathrm{MWh}$.

- With the penalty contract, penalties are applied to under- as well as overconsumption. A penalty of $\$ 50 / \mathrm{MWh}$ has to be paid if the daily electricity purchase from the penalty contract is below $20 \mathrm{MWh}$ or exceeds $80 \mathrm{MWh}$. Otherwise, there is no cost in addition to the base cost.

Moreover, when applied, the maximum amount that can be purchased from each contract every hour is $10 \mathrm{MWh}$. 
Using the data from the illustrative example, we now consider five cases which differ in the product demand and the applied power contracts as shown in Table 6. In all cases, all processes are in the on mode at the start of the scheduling horizon. Also, it is assumed that no mode switching has occurred in the previous 12 hours. The total electricity costs in each case obtained by solving the MILP model are listed in the last column of Table 6.

Table 6: The table lists the five cases, which differ in the product demands and in the power contracts, with the optimal total electricity costs.

\begin{tabular}{cccc}
\hline Case & Product Demand & Power Contracts & Total Electricity Cost \\
\hline 1 & low & TOU & $\$ 5,771$ \\
2 & high & TOU & $\$ 14,624$ \\
3 & medium & TOU & $\$ 11,697$ \\
4 & medium & TOU, discount & $\$ 11,057$ \\
5 & medium & TOU, penalty & $\$ 11,346$ \\
\hline
\end{tabular}

For Case 1, the optimal schedule is shown in Figure 10, which depicts a Gantt chart showing the operating modes of each process in each time period. Figure 11 shows the amount of electricity consumed by each process. Also, one can observe that the plant is shut down for a large portion of the time, primarily when the electricity price is high. This large shift in load is possible due to the low demand and the flexibility in the inventory as indicated by the changes in inventory levels shown in Figure 12. Also note that for the conversion of A to B, Process 1.1 is preferred over Process 1.2 due to its higher capacity and lower unit electricity consumption. Similarly, Process 4.2 is preferred over Process 4.1 due to its higher efficiency.

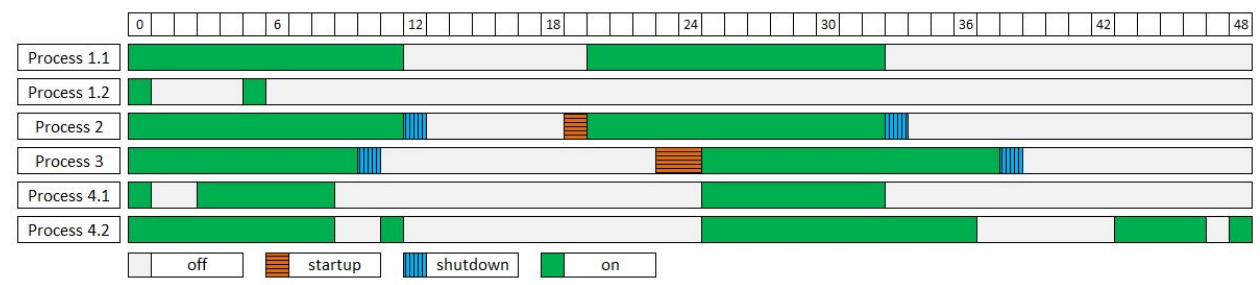

Figure 10: Gantt chart for the optimal schedule in Case 1. Selected operating modes of each process are shown for each time period. 


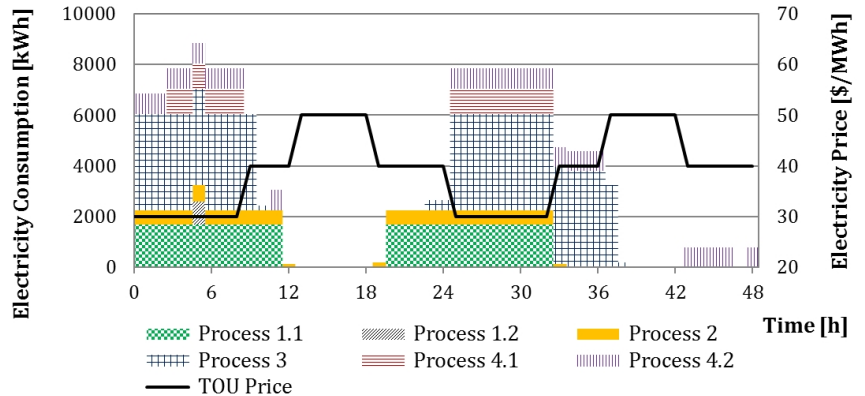

Figure 11: Amount of electricity consumed by each process in Case 1.

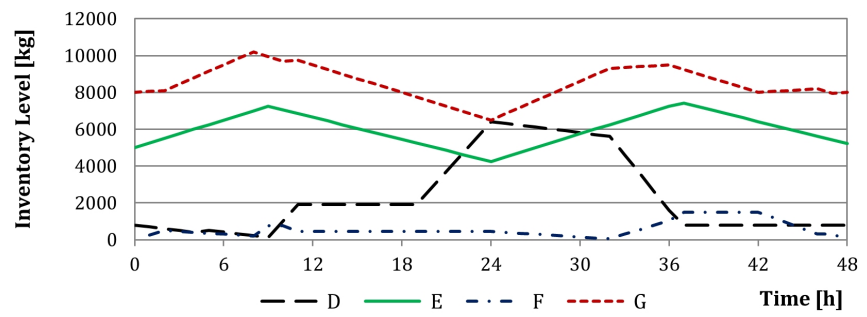

Figure 12: Inventory profiles of the intermediate materials $\mathrm{D}$ and $\mathrm{F}$, and the final products $\mathrm{E}$ and $\mathrm{G}$ in Case 1.

The electricity consumption profiles in Case 2 are shown in Figure 13, which only expresses moderate load shifting. This can be explained by the reduced level of process flexibility due to the high demand. Here, the plant has to utilize almost its entire production capacity to satisfy demand. Figure 14 shows the result for Case 3, which indicates that with medium time-varying demand, there is again significant potential for load shifting.

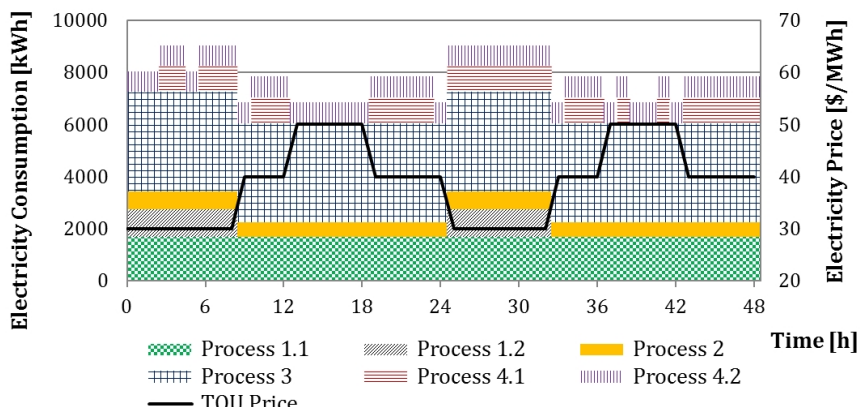

Figure 13: Amount of electricity consumed by each process in Case 2 . 


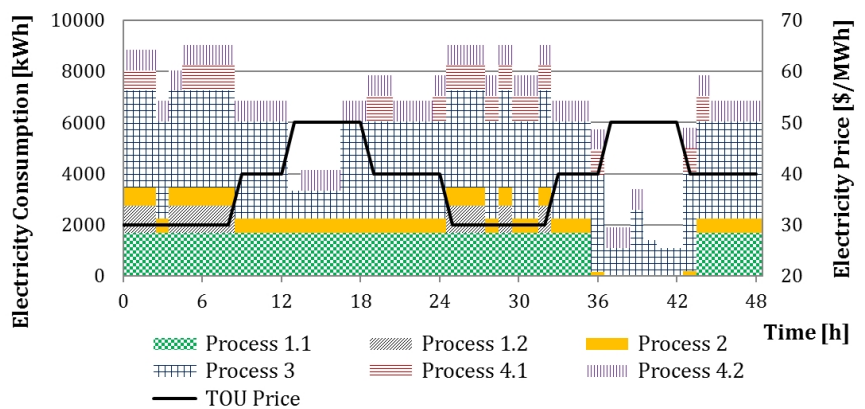

Figure 14: Amount of electricity consumed by each process in Case 3 .

In Case 4, the discount contract is applied in addition to the TOU contract. Figure 15 shows the breakdown of the total electricity purchase into the purchases from these two contracts and the corresponding base prices. The majority of the consumed electricity in the first 24 hours, namely $135.5 \mathrm{MWh}$, is purchased from the discount contract. By doing so, we are taking advantage of the best possible discount. However, there are still time periods in which the TOU contract offers a lower price. In those hours, electricity is purchased from the TOU contract. On the second day, 53.9 MWh are purchased from the discount contract. We would purchase more from the discount contract for a larger discount; however, the threshold for that discount is too high for this to be beneficial. Therefore, the rest of the consumed electricity is purchased from the TOU contract.

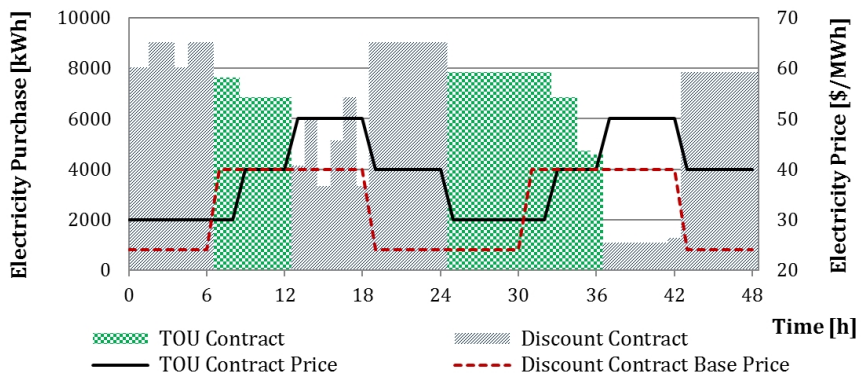

Figure 15: Breakdown of the total electricity purchase into the purchases from the TOU and the discount contracts in Case 4.

In Case 5, the TOU and the penalty contracts are applied. Figure 16 shows the breakdown of the total electricity purchase and the base prices. Notice that the price for the penalty contract is higher on the second day. $80 \mathrm{MWh}$ and $20 \mathrm{MWh}$ are purchased from the penalty contract on the first and second day, respectively. It is clear that because of the low price on the first day, we try to purchase as much as possible from the penalty contract without having to pay 
any penalties. On the second day, since the price is now high, we purchase just enough to avoid penalties.

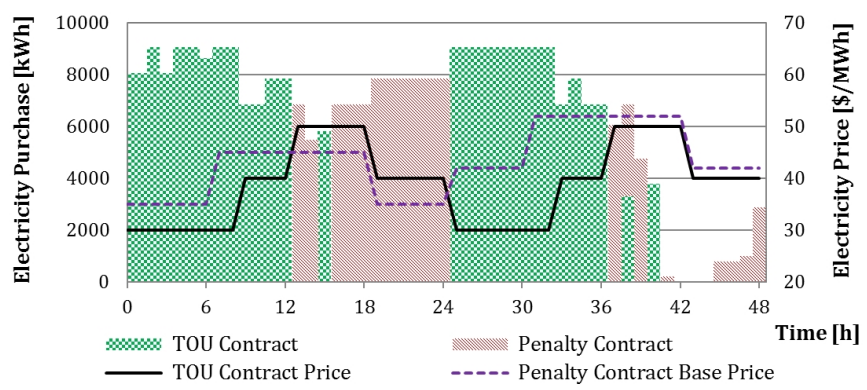

Figure 16: Breakdown of the total electricity purchase into the purchases from the TOU and the penalty contracts in Case 5.

All models were implemented in GAMS 24.4.1 (GAMS Development Corporation, 2015), and the commercial solver CPLEX 12.6.1 was applied to solve the MILPs. In each case, the model has approximately 5,000 continuous variables, 2,500 binary variables, and 7,500 constraints. All models were solved to zero integrality gap in less than 20 seconds wall-clock time on an Intel ${ }^{\circledR}$ Core $^{\mathrm{TM}}$ i7-2600 machine at $3.40 \mathrm{GHz}$ with eight processors and $8 \mathrm{~GB}$ RAM running Windows 7 Professional.

\section{Industrial case study}

We now apply the proposed model to a real-world industrial case study provided by Praxair. Here, we consider an air separation plant that produces gaseous oxygen (GO2), gaseous nitrogen (GN2), liquid oxygen (LO2), liquid nitrogen (LN2), and liquid argon (LAr). The corresponding process network is shown in Figure 17. The GO2, LO2, LN2, and LAr flowing out of the air separation (AS) process can be directly sold whereas GN2 has to be further compressed before it can be supplied to the customers. Two kinds of GN2 are sold: medium-pressure GN2 (MPGN2) and high-pressure GN2 (HPGN2). GN2 is compressed to MPGN2 through Process LMCompGN2 and can be further compressed to HPGN2 through Process MHCompGN2; it can also be directly converted to HPGN2 by running Process LHCompGN2. Furthermore, GN2 can be liquefied to LN2 by running Process LiqGN2. Overproduced gaseous products can be vented through a venting process, and all liquid products can be converted into the corresponding gaseous products through a so-called driox process. The CRS models for each process have been generated by applying the data-driven algorithm proposed Zhang et al. (2015b) to real process data.

While there are inventories of the liquid products, we assume that gaseous products cannot be stored. Electricity can be purchased from three different 


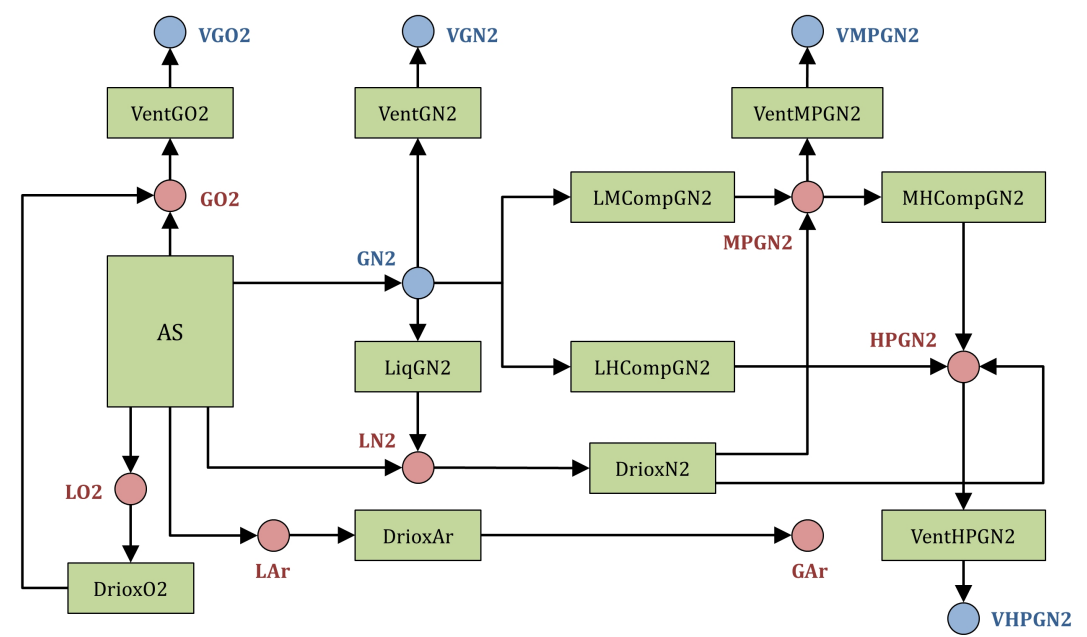

Figure 17: Process network representing the given air separation plant.

sources: a TOU contract, a penalty contract, and the spot market. While preagreed prices for the TOU and the penalty contracts are known, a forecast of the hourly-varying spot price is used in the model. For the penalty contract, penalties are only paid for underconsumption.

The scheduling horizon is one week, to which an hourly time discretization is applied resulting in 168 time periods. Demand profiles for all products are given. Note that while there is continuous demand for gaseous products, the demand for each liquid product is assumed to occur only at the end of each day. The assumption here is that there is sufficient capacity in the inventory to handle flows into and out of the inventory tank throughout the day. The demand at the end of the day is then the total amount of product that needs to be drawn from the tank over the course of the day.

We solve the resulting MILP model to obtain the optimal schedule. Note that due to confidentiality reasons, we cannot disclose information about the plant specifications as well as the actual product demand. Therefore, all results presented in the following are given without units and the values are normalized if necessary. However, the analysis and interpretation of the results are still meaningful.

Figure 18 shows the electricity consumption profiles for each of the processes. The vast majority of the electricity consumption is attributed to the air separation unit (ASU). The GN2 liquefier also consumes a large amount of electricity but is only used five times, each time for a few hours. Compared to the ASU and the GN2 liquefier, the pipeline compressors contribute relatively little to the total electricity consumption. Significant load shifting can be observed in the schedule; this is mainly realized by operating the liquefier during low-price hours, which allows a fairly constant operation of the other processes.

The electricity prices for the TOU and the penalty contracts as well as 


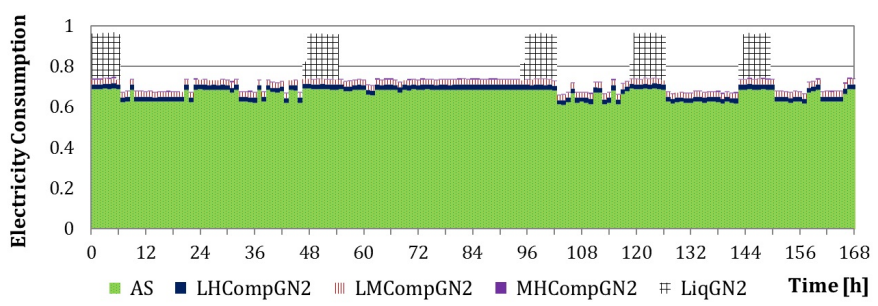

Figure 18: Amount of electricity consumed by each process of the air separation plant.

the spot electricity price are shown in Figure 19. The breakdown of the total electricity purchase into the purchases from the three different sources is shown in Figure 20. One can observe that in each time period, we choose to purchase from the source with the lowest price. Two sources are chosen in the same time period only when the maximum purchase amount is reached for one of the two sources. Also, sufficient amount of electricity is purchased from the penalty contract such that no penalty has to be paid.

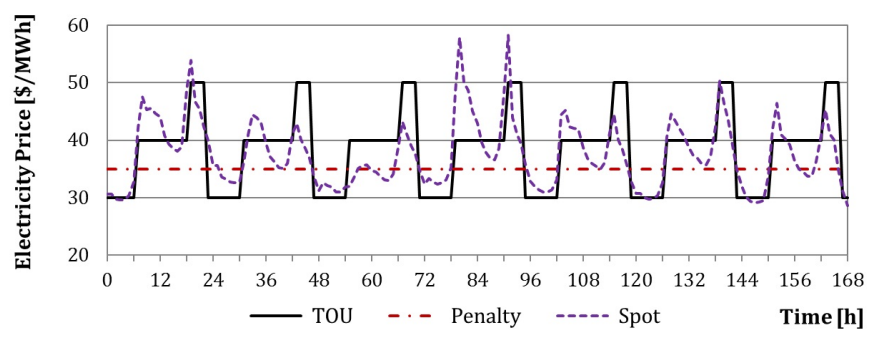

Figure 19: Electricity prices for the TOU contract, the penalty contract, and the spot market.

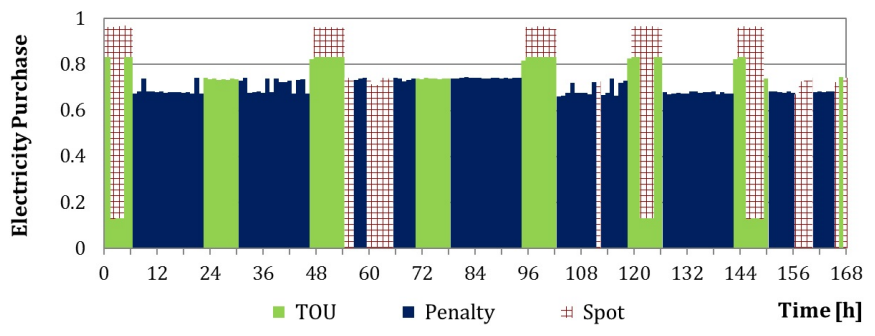

Figure 20: Breakdown of the total electricity purchase into the purchases from the three difference sources.

As an example, Figure 21 shows the amount of GN2 produced by the AS process and the GN2 flows into the subsequent processes. Large portion of GN2 
is compressed to feed the product pipelines. Another significant amount of GN2 is liquefied to increase the production of LN2. However, the majority of GN2 is vented. The reason for this overproduction of GN2 is that the plant is "oxygenlimited", which means that the production is driven by the oxygen demand. At such a plant, the nitrogen production usually exceeds the demand, which makes venting of GN2 necessary.

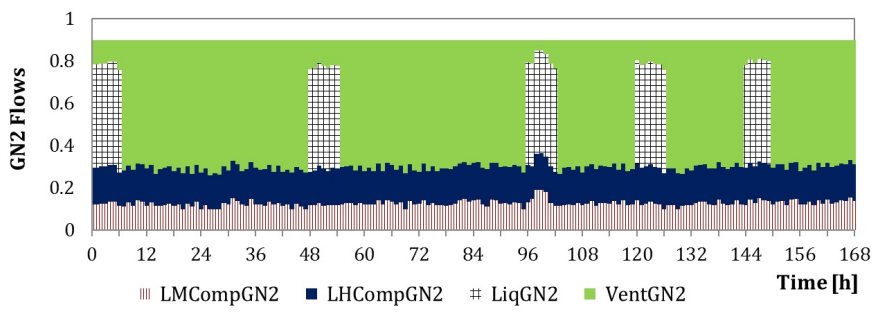

Figure 21: GN2 production and its breakdown into feeds for different processes.

Figure 22 shows the amounts of LN2 produced by the ASU and the GN2 liquefier in each time period. The inventory increases when LN2 is produced and it decreases when LN2 is drawn from the tank to satisfy demand, which occurs every 24 hours and is not shown in the figure. One can see that in addition to the production through the AS process, a large amount of LN2 is obtained by liquefying GN2. In this solution, driox is not used in any of the time periods.

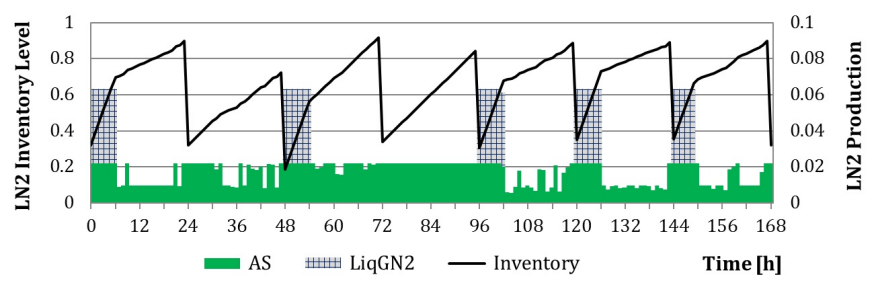

Figure 22: LN2 production and inventory profile.

The MILP model for the industrial-scale problem has 28,808 continuous variables, 9,776 binary variables, and 113,810 constraints. It was solved to zero integrality gap in 7 seconds wall-clock time on the same Intel ${ }^{\circledR}$ Core $^{\mathrm{TM}}$ i7-2600 machine used to solve the illustrative example. A brief computational study was conducted by solving several instances of the problem with different input parameters. Almost all instances were solved within two minutes.

\section{Conclusions}

In this work, a general discrete-time MILP model has been developed for the scheduling of continuous power-intensive process networks with various power 
contracts. The main focus of the proposed formulation are the accurate and efficient representation of the operational flexibility in the production process as well as the modeling of power contracts. A process network has been considered where each process is represented by a Convex Region Surrogate model. A mode-based formulation has been adopted to model operational transitions. Moreover, a block formulation has been proposed that allows the modeling of a large variety of power contracts including commonly occurring discount and penalty contracts.

The proposed scheduling model has been applied to an illustrative example as well as to a real-world air separation plant. The results demonstrate the capability of the model in representing the operation of continuous process networks and the flexibility in modeling different power contracts. This allows the optimal scheduling of power-intensive processes involving load shifting within the feasible range of operation. Furthermore, the proposed MILP model has proven to be computationally very efficient. Large-scale problems with tens of thousands of variables and hundreds of thousands of constraints can be solved within a few minutes, which allows the use of such a scheduling tool in a real industrial setting.

\section{Nomenclature}

$\begin{array}{ll}\text { Indices } & \\ & \\ b, b^{\prime} & \text { contract blocks } \\ c & \text { power contracts } \\ i & \text { processes } \\ j & \text { materials } \\ l & \text { vertices } \\ m, m^{\prime}, m^{\prime \prime} & \text { operating modes } \\ r & \text { (convex) operating subregions } \\ t & \text { time periods }\end{array}$

Sets

$B_{c} \quad$ contract blocks for block contract $c$

$C \quad$ power contracts

$\bar{C} \quad$ block contracts, i.e. discount and penalty contracts, $\bar{C} \subseteq C$

I processes

$\bar{I}_{j} \quad$ processes receiving material $j$

$\hat{I}_{j} \quad$ processes producing material $j$

$J_{i} \quad$ materials associated with process $i, J_{i}=\bar{J}_{i} \cup \hat{J}_{i}$

$\bar{J}_{i} \quad$ materials that are inputs to process $i$

$\hat{J}_{i} \quad$ materials that are outputs of process $i$

$L_{i m r} \quad$ vertices of subregion $r$ in mode $m$ of process $i$

$M_{i} \quad$ modes for process $i$ 
$R_{i m} \quad$ subregions in mode $m$ of process $i$

$S Q_{i} \quad$ predefined sequences of mode transitions in process $i$

$T \quad$ time periods, $T=\left\{-\theta^{\max }+1,-\theta^{\max }+2, \ldots, 0,1, \ldots, t^{\text {fin }}\right\}$

$\bar{T} \quad$ time periods in the scheduling horizon, $\bar{T}=\left\{1,2, \ldots, t^{\mathrm{fin}}\right\}$

$\widehat{T}_{c} \quad$ cumulative electricity consumption meter reading times for contract $c$

$T R_{i} \quad$ possible mode transitions in process $i$

$T R_{i m}^{\mathrm{f}} \quad$ modes of process $i$ from which mode $m$ can be directly reached

$T R_{i m}^{\mathrm{t}} \quad$ modes of process $i$ which can be directly reached from mode $m$

\section{Parameters}

$D_{j t} \quad$ demand for material $j$ in time period $t[\mathrm{~kg}]$

$E_{c t}^{\min }$ minimum amount of electricity that has to be purchased from contract $c$ in time period $t[\mathrm{kWh}]$

$E_{c t}^{\max } \quad$ maximum amount of electricity that can be purchased from contract $c$ in time period $t[\mathrm{kWh}]$

$H_{c b}^{\max } \quad$ maximum cumulative electricity purchase in block $b$ of contract $c[\mathrm{kWh}]$

$Q_{j}^{\mathrm{fin}} \quad$ minimum final inventory level for material $j[\mathrm{~kg}]$

$Q_{j}^{\text {ini }} \quad$ initial inventory level for material $j[\mathrm{~kg}]$

$Q_{j}^{\min } \quad$ minimum inventory level for material $j[\mathrm{~kg}]$

$Q_{j}^{\max } \quad$ maximum inventory level for material $j[\mathrm{~kg}]$

$t^{\text {fin }} \quad$ index of the last time period of the scheduling horizon $\bar{T}$

$W_{j}^{\max } \quad$ maximum purchasing amount for material $j[\mathrm{~kg}]$

$y_{i m}^{\text {ini }} \quad 1$ if process $i$ was operating in mode $m$ in time period 0

$z_{i m m^{\prime} t}^{\text {ini }} \quad 1$ if operation of process $i$ switched from mode $m$ to mode $m^{\prime}$ at time $t$ before time 0

$\alpha_{c t} \quad$ base unit price for electricity purchased from contract $c$ in time period $t[\$ / \mathrm{kWh}]$

$\beta_{c b t} \quad$ additional unit price for cumulative electricity purchased from block $b$ of contract $c$ at time $t[\$ / \mathrm{kWh}]$

$\gamma_{i m r j} \quad$ unit electricity consumption corresponding to material $j$ if process $i$ operates in subregion $r$ of mode $m[\mathrm{kWh} / \mathrm{kg}]$

$\delta_{i m r} \quad$ fixed electricity consumption if process $i$ operates in subregion $r$ of mode $m[\mathrm{kWh}]$

$\Delta_{i m j}^{\max } \quad$ maximum rate of change in the amount of material $j$ consumed or produced in mode $m$ of process $i[\mathrm{~kg}]$

$\Delta t \quad$ length of one time period $[\mathrm{h}]$

$\zeta_{c t}^{\mathrm{o}} \quad$ unit overconsumption penalty cost for contract $c$ at time $t[\$ / \mathrm{kWh}]$

$\zeta_{c t}^{\mathrm{u}} \quad$ unit underconsumption penalty cost for contract $c$ at time $t[\$ / \mathrm{kWh}]$

$\theta_{i m m^{\prime}} \quad$ minimum stay time in mode $m^{\prime}$ after switching from mode $m$ to mode $m^{\prime}$ in process $i[\Delta t]$

$\theta^{\max } \quad$ largest minimum stay time $[\Delta t]$

$\bar{\theta}_{i m m^{\prime} m^{\prime \prime}} \quad$ fixed stay time in mode $m^{\prime}$ in the predefined sequence $\left(m, m^{\prime}, m^{\prime \prime}\right)$ in process $i[\Delta t]$

$\phi_{i m r l j} \quad$ amount of material $j$ associated with vertex $l$ of subregion $r$ in mode $m$ of process $i[\mathrm{~kg}]$ 


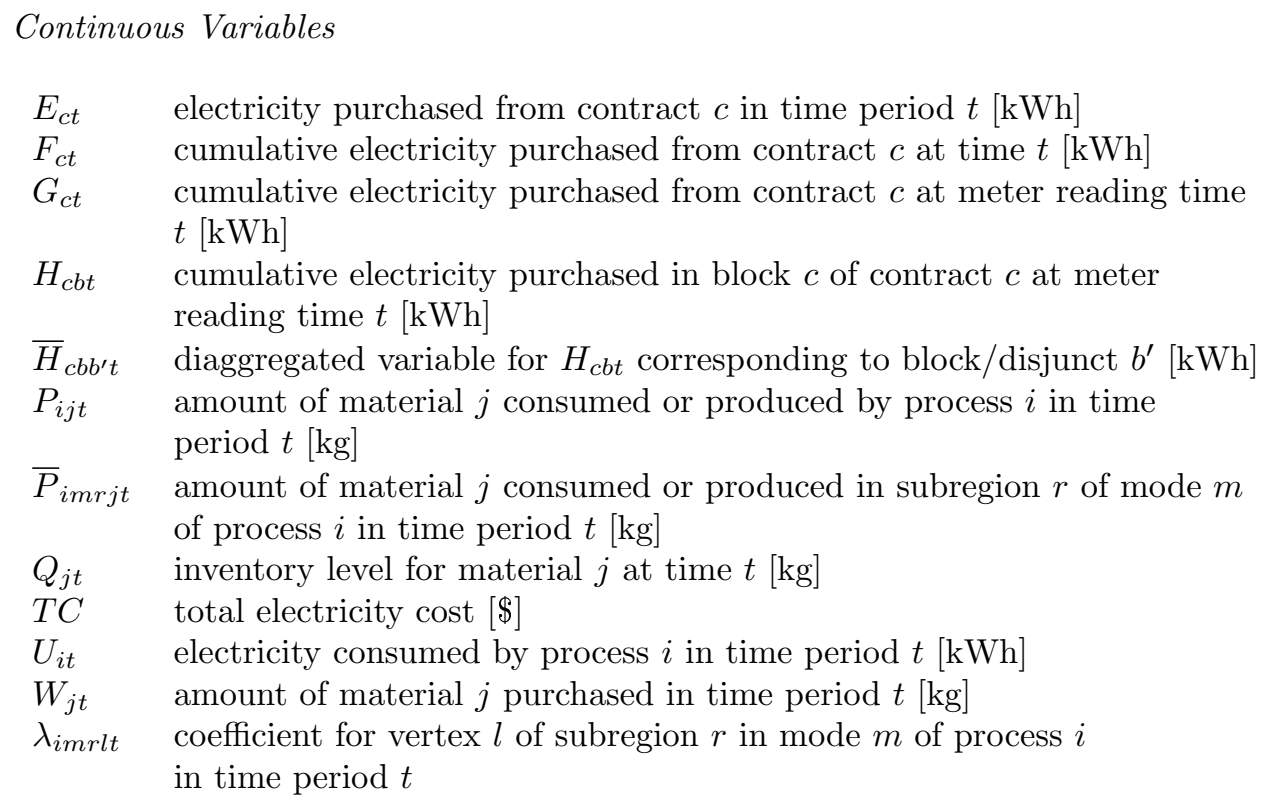

Binary Variables

$x_{c b t} \quad 1$ if block $b$ is the highest block reached for contract $c$ at time $t$

$y_{\text {imt }} \quad 1$ if process $i$ operates in mode $m$ in time period $t$

$\bar{y}_{i m r t} \quad 1$ if process $i$ operates in subregion $r$ of mode $m$ in time period $t$

$z_{i m m^{\prime} t} \quad 1$ if process $i$ switches from mode $m$ to mode $m^{\prime}$ at time $t$

\section{Boolean Variables}

$X_{c b t}$ true if block $b$ is the highest block reached for contract $c$ at time $t$

$Y_{i m t} \quad$ true if process $i$ operates in mode $m$ in time period $t$

$\bar{Y}_{i m r t}$ true if process $i$ operates in subregion $r$ of mode $m$ in time period $t$

\section{Acknowledgements}

The authors gratefully acknowledge the financial support from the National Science Foundation under Grant No. 1159443 and from Praxair.

\section{References}

Albadi, M. H., El-Saadany, E. F., 2008. A summary of demand response in electricity markets. Electric Power Systems Research 78 (11), 1989-1996.

Alle, A., Papageorgiou, L. G., Pinto, J. M., 2004. A mathematical programming approach for cyclic production and cleaning scheduling of multistage continuous plants. Computers and Chemical Engineering 28 (1-2), 3-15. 
Ashok, S., 2006. Peak-load management in steel plants. Applied Energy 83 (5), 413-424.

Babu, C. A., Ashok, S., 2008. Peak Load Management in Electrolytic Process Industries. IEEE Transactions on Power Systems 23 (2), 399-405.

Balas, E., 1985. Disjunctive Programming and a Hierarchy of Relaxations for Discrete Optimization Problems. SIAM Journal on Algebraic Discrete Methods 6 (3), 466-486.

Castro, P. M., Barbosa-Póvoa, A. P., Matos, H. A., Novais, A. Q., 2004. Simple Continuous-Time Formulation for Short-Term Scheduling of Batch and Continuous Processes. Industrial \& Engineering Chemistry Research 43, 105-118.

Castro, P. M., Harjunkoski, I., Grossmann, I. E., 2009. New Continuous-Time Scheduling Formulation for Continuous Plants under Variable Electricity Cost. Industrial \& Engineering Chemistry Research 48 (14), 6701-6714.

Castro, P. M., Harjunkoski, I., Grossmann, I. E., 2011. Optimal scheduling of continuous plants with energy constraints. Computers \& Chemical Engineering 35 (2), 372-387.

Castro, P. M., Sun, L., Harjunkoski, I., 2013. Resource-Task Network Formulations for Industrial Demand Side Management of a Steel Plant. Industrial \& Engineering Chemistry Research 52, 13046-13058.

Charles River Assosicates, 2005. Primer on Demand-Side Management. Tech. Rep. February, The World Bank.

Floudas, C. A., Lin, X., 2004. Continuous-time versus discrete-time approaches for scheduling of chemical processes: a review. Computers \& Chemical Engineering 28 (11), 2109-2129.

GAMS Development Corporation, 2015. GAMS version 24.4.1.

Giannelos, N. F., Georgiadis, M. C., 2002. A Simple New Continuous-Time Formulation for Short-Term Scheduling of Multipurpose Batch Processes. Industrial \& Engineering Chemistry Research 41, 2178-2184.

Harjunkoski, I., Maravelias, C. T., Bongers, P., Castro, P. M., Engell, S., Grossmann, I. E., Hooker, J., Méndez, C., Sand, G., Wassick, J., 2014. Scope for industrial applications of production scheduling models and solution methods. Computers \& Chemical Engineering 62, 161-193.

Ierapetritou, M. G., Floudas, C. A., 1998. Effective Continuous-Time Formulation for Short-Term Scheduling. 2. Continuous and Semicontinuous Processes. Industrial \& Engineering Chemistry Research 37 (11), 4360-4374. 
Ierapetritou, M. G., Wu, D., Vin, J., Sweeney, P., Chigirinskiy, M., 2002. Cost Minimization in an Energy-Intensive Plant Using Mathematical Programming Approaches. Industrial \& Engineering Chemistry Research 41 (21), 52625277 .

Karimi, I. A., McDonald, C. M., 1997. Planning and scheduling of parallel semicontinuous processes. 2. Short-term scheduling. Industrial and Engineering Chemistry Research 36 (7), 2701.

Karwan, M. H., Keblis, M. F., 2007. Operations planning with real time pricing of a primary input. Computers \& Operations Research 34 (3), 848-867.

Kondili, E., Pantelides, C. C., Sargent, R. W. H., 1993. A General Algorithm for Short-Term Scheduling of Batch Operations - I. MILP Formulation. Computers \& Chemical Engineering 17 (2), 211-227.

Kopanos, G. M., Puigjaner, L., Maravelias, C. T., 2011. Production Planning and Scheduling of Parallel Continuous Processes with Product Families. Industrial \& Engineering Chemistry Research 50 (3), 1369-1378.

Maravelias, C. T., 2012. General Framework and Modeling Approach Classification for Chemical Production Scheduling. AIChE Journal 58 (6), 1812-1828.

Méndez, C. A., Cerdá, J., Grossmann, I. E., Harjunkoski, I., Fahl, M., 2006. State-of-the-art review of optimization methods for short-term scheduling of batch processes. Computers \& Chemical Engineering 30 (6-7), 913-946.

Merkert, L., Harjunkoski, I., Isaksson, A., Säynevirta, S., Saarela, A., Sand, G., 2014. Scheduling and energy - Industrial challenges and opportunities. Computers \& Chemical Engineering 72, 183-198.

Mitra, S., Grossmann, I. E., Pinto, J. M., Arora, N., 2012. Optimal production planning under time-sensitive electricity prices for continuous power-intensive processes. Computers \& Chemical Engineering 38, 171-184.

Mitra, S., Sun, L., Grossmann, I. E., 2013. Optimal scheduling of industrial combined heat and power plants under time-sensitive electricity prices. Energy $54,194-211$.

Pantelides, C. C., 1994. Unified Frameworks for Optimal Process Planning and Scheduling. In: Foundations of computer-aided process operations. New York, pp. $253-274$.

Paulus, M., Borggrefe, F., 2011. The potential of demand-side management in energy-intensive industries for electricity markets in Germany. Applied Energy 88 (2), 432-441.

Pinto, J. M., Grossmann, I. E., 1994. Optimal cyclic scheduling of multistage continuous multiproduct plants. Computers \& Chemical Engineering 18 (9), 797-816. 
Sahinidis, N. V., Grossmann, I. E., 1991. MINLP model for cyclic multiproduct scheduling on continuous parallel lines. Computers \& Chemical Engineering 15 (2), 85-103.

Samad, T., Kiliccote, S., 2012. Smart grid technologies and applications for the industrial sector. Computers \& Chemical Engineering 47, 76-84.

Schilling, G., Pantelides, C. C., 1996. A simple continuous-time process scheduling formulation and a novel solution approach. Computers \& Chemical Engineering 20 (96), 1221-1226.

Shah, N., Pantelides, C., Sargent, R., 1993. A general algorithm for shortterm scheduling of batch operations - II. Computational issues. Computers \& Chemical Engineering 17 (2), 229-244.

Shaik, M. A., Floudas, C. A., 2007. Improved unit-specific event-based continuous-time model for short-term scheduling of continuous processes: Rigorous treatment of storage requirements. Industrial and Engineering Chemistry Research 46 (6), 1764-1779.

Sundaramoorthy, A., Maravelias, C. T., 2011. Computational Study of NetworkBased Mixed-Integer Programming Approaches for Chemical Production Scheduling. Industrial \& Engineering Chemistry Research 50, 5023-5040.

Sung, C., Maravelias, C. T., 2009. A Projection-Based Method for Production Planning of Multiproduct Facilities. AIChE Journal 55 (10), 2614-2630.

Vujanic, R., Mariéthos, S., Goulart, P., Morari, M., 2012. Robust Integer Optimization and Scheduling Problems for Large Electricity Consumers. In: Proceedings of the 2012 American Control Conference. pp. 3108-3113.

Zhang, Q., Grossmann, I. E., 2015. Planning and Scheduling for Industrial Demand Side Management: Advances and Challenges. To appear in Alternative Energy Sources and Technologies: Process Design and Operation.

Zhang, Q., Grossmann, I. E., Heuberger, C. F., Sundaramoorthy, A., Pinto, J. M., 2015a. Air Separation with Cryogenic Energy Storage: Optimal Scheduling Considering Electric Energy and Reserve Markets. AIChE Journal 61 (5), 1547-1558.

Zhang, Q., Grossmann, I. E., Sundaramoorthy, A., Pinto, J. M., 2015b. Datadriven construction of Convex Region Surrogate models. To appear in Optimization and Engineering.

Zhang, X., Sargent, R. W. H., 1996. The optimal operation of mixed production facilitiesa general formulation and some approaches for the solution. Computers \& Chemical Engineering 20 (6-7), 897-904. 